\title{
Therapeutic Monitoring of Adalimumab at Non- Trough Levels to Gauge Its Efficacy in Patients with Inflammatory Bowel Disease in Clinical Practice
}

\section{Kato Masaichi}

Hamamatsu South Hospital

Ken Sugimoto ( $\nabla$ sugimken@hama-med.ac.jp )

Hamamatsu University School of Medicine https://orcid.org/0000-0001-9586-1097

Ikeya Kentaro

Hamamatsu South Hospital

Takano Ryosuke

Hamamatsu South Hospital

Matsuura Ai

Hamamatsu South Hospital

Takahiro Miyazu

Hamamatsu Ika Daigaku

Natsuki Ishida

Hamamatsu Ika Daigaku

Satoshi Tamura

Hamamatsu Ika Daigaku

Shinya Tani

Hamamatsu Ika Daigaku

Mihoko Yamade

Hamamatsu Ika Daigaku

Yasushi Hamaya

Hamamatsu Ika Daigaku

Moriya Iwaizumi

Hamamatsu Ika Daigaku

\section{Satoshi Osawa}

Hamamatsu Ika Daigaku

\section{Takahisa Furuta}

Hamamatsu Ika Daigaku

Hanai Hiroyuki

Hamamatsu South Hospital 


\section{Research article}

Keywords: adalimumab, tumor-necrosis factor-alpha, anti-drug antibody, endoscopic remission, immunomodulator

Posted Date: October 6th, 2020

DOI: https://doi.org/10.21203/rs.3.rs-79319/v1

License: (c) (i) This work is licensed under a Creative Commons Attribution 4.0 International License.

Read Full License 


\section{Abstract}

Background: Adalimumab (ADA) trough level and anti-ADA antibody (AAA) positivity could influence mucosal healing and loss of response in patients with inflammatory bowel disease (IBD). This study aimed to clarify the correlation between ADA monitoring, including non-trough level and real-world IBD clinical outcomes.

Methods: This retrospective, observational, single-center study included 19 patients with ulcerative colitis (UC) and 33 patients with Crohn's disease (CD) treated with ADA from January 2007 to August 2018. Serum ADA and AAA levels were measured 4-14 days after ADA administration.

Results: The AAA positivity rate was $23.1 \%(12 / 52)$. The ADA continuity was higher in the AAA-negative group than in the AAA-positive group $(P=0.223)$. Receiver operating characteristic $(\mathrm{ROC})$ analysis revealed that a serum AAA cut-off value of $9.2 \mu \mathrm{g} / \mathrm{mL}$ was associated with ADA continuity (area under the curve [AUC]: $0.767,95 \%$ confidence interval [CI]: 0.636-0.899). The ADA level was significantly higher in the endoscopic remission group than in the non-remission group (12.4 vs. $6.4 \mu \mathrm{g} / \mathrm{mL}, P=0.02)$. Based on the ROC curve analysis results of serum ADA level and endoscopic remission, the cut-off value of serum ADA level was set to $11.1 \mu \mathrm{g} / \mathrm{mL}$ (AUC: $0.716,95 \% \mathrm{Cl}$ : $0.533-0.900$ ).

Conclusions: Regardless of infliximab administration history, under combined use of ADA with immunomodulators and AAA positivity, ADA continuity was significantly higher when the serum AAA level 4-14 days after ADA administration was $\geq 9.2 \mu \mathrm{g} / \mathrm{mL}$. Furthermore, endoscopic remission can be expected with a serum ADA level of $\geq 11.1 \mu \mathrm{g} / \mathrm{mL}$.

\section{Background}

Although the etiology of refractory inflammatory lesions in the intestinal tract of patients with inflammatory bowel diseases (IBDs) such as ulcerative colitis (UC) and Crohn's disease (CD) is unknown, inflammation caused by immune system dysregulation in the intestinal mucosa has been postulated to be the underlying cause of IBD pathogenesis [1]. The mainstay treatment for IBD is the suppression of immunocompetent cells by the systemic administration of steroids [2]. However, during recent years, biologics targeting cytokines produced by immunocompetent cells have been increasingly used in IBD treatment [3]. Among the biologics, anti-TNFa agents are widely used for IBD. In Japan, infliximab (IFX) and adalimumab (ADA) are approved for CD treatment and golimumab is approved for UC treatment.

Biologics such as IFX and ADA not only induce clinical remission in patients with IBD but also cause mucosal healing [4-6]. However, loss of response (LOR) is a major concern in IBD management, as it occurs within 1 year in an estimated $13 \%$ of patients treated with IFX [7] and $20 \%$ of patients treated with ADA [8]. Although the mechanism of LOR is not clear, immunogenicity against anti-TNFa inhibitors, manifested as a decrease in serum level (trough level) of anti-TNFa owing to the production of anti-drug antibodies or undetectability of anti-TNFa, is considered to be involved in LOR [5]. 
The importance of maintaining a high trough level of anti-TNFa agents has been shown in previous studies, which demonstrated that a low IFX trough level makes it difficult to achieve mucosal healing [4, 5 , 9]. As reported, the risk of LOR in patients with antibodies against IFX (ATI) is three times that of patients without ATI [10]; it is important to suppress the production of anti-drug antibodies. Both trough level and anti-ADA antibody (AAA) have been associated with mucosal healing and LOR [11]. During recent years, therapeutic drug monitoring (TDM), a strategy to optimize treatment by measuring the serum trough level and anti-drug antibody level, has been recommended to maximize the effects of these biological agents [12]. Therapeutic drug monitoring is of two types: reactive TDM, which promotes decision making for patients who show treatment failure, and proactive TDM, which optimizes treatment and potentially avoids flare and LOR in patients who have achieved some therapeutic effect [13]. However, the treat-to-target strategy, which has been advocated during recent years, emphasizes the importance of proactive TDM in the treatment of patients with IBD in clinical remission [14]. A review by Mitrev et al. recommends steady-state trough levels between 3 and $8 \mu \mathrm{g} / \mathrm{mL}$ for IFX and between 5 and $12 \mu \mathrm{g} / \mathrm{mL}$ for ADA [12]. The upper limit of the therapeutic window of ADA trough level to achieve clinical remission in patients with IBD has not been defined.

Another problem in real-world clinical practice of TDM is the regular visit dates of patients do not always coincide with the trough measurement date, i.e. 14 days after ADA administration. It is not always possible to schedule another consultation visit only for the measurement of trough or to change the time of injection to measure the trough level during the consultation visit. Interestingly, Ward et al. reported that the trough level could be estimated from serum ADA level from days 3 to 9 after ADA injection, suggesting the feasibility of TDM even without trough level measurement of ADA [15]. In addition, authors reported that 8 weeks after the initiation of ADA therapy, the average fluctuation in ADA level during the 2 weeks between successive administrations of ADA was approximately $3 \mu \mathrm{g} / \mathrm{mL}$ [16]. Therefore, the purpose of our study was to analyze the effects of serum ADA levels, including its nontrough levels, and AAA presence on subsequent treatment outcomes in patients with IBD receiving ADA. We also aimed to clarify the correlation between TDM for ADA and clinical outcomes in the real-world setting.

\section{Methods}

\section{Patients}

Fifty-two patients with IBD (UC or CD) were enrolled in this study and treated with ADA at Hamamatsu South Hospital, Hamamatsu, Japan, from January 2007 to August 2018. Serum drug (ADA) and AAA levels were measured during TDM. All patients provided informed consent before enrolling in this study. Patients with Behcet's disease, indeterminate colitis, and unclassified IBD were excluded from the study. Adalimumab was prescribed at standard doses of $160 \mathrm{mg}$ at week $0,80 \mathrm{mg}$ at week 2, and $40 \mathrm{mg}$ subsequently every other week for maintenance; the drug was administered subcutaneously. The observation period was from the start of ADA administration to ADA discontinuation, and observations were continued until August 2018 in patients who were still on ADA in August 2018. We measured the 
anti-drug antibody and drug levels at patients' visit dates; we excluded patients whose antibody and drug levels were measured within 3 days of ADA administration.

\section{Study design}

This was a retrospective, observational single-center study. The primary endpoints of this study were the comparison of treatment continuity between (i) the AAA-positive and -negative groups and (ii) the highand low-serum ADA groups. The secondary endpoints were (i) comparison of endoscopic remission rate between patients with high- and low-serum ADA levels and (ii) extraction of factors that may influence serum ADA level. Treatment continuity implied that neither the dose nor the dosing interval of ADA was changed during the observation period. Accordingly, patients who discontinued ADA therapy or received treatment enhancements, such as double-dose administration or shortened ADA administration period, did not fit this description. Discontinuation of ADA because of transferring patients to another hospital, the preference of patients, or discontinuation because of adverse events were not considered "censored." Endoscopic remission was defined as a Mayo endoscopic score of $\leq 1$ in patients with UC and a simple endoscopic score for $C D$ of $\leq 3$ in patients with $C D$. Endoscopic evaluation was conducted only for patients who underwent endoscopy after introducing ADA.

\section{Laboratory methods}

We measured the serum level of ADA and AAA (free and bound) using enzyme-linked immunosorbent assays (ELISAs) (IDKmonitor ${ }^{\circledR}$ Adalimumab drug level ELISA and IDKmonitor ${ }^{\circledR}$ Adalimumab total ADA ELISA, Immundiagnostik AG, Germany).

\section{Statistical analysis}

The Kaplan-Meier estimator was used to generate survival curves for the AAA-positive and -negative groups. The log-rank test was used to compare persistence rate (treatment continuity) with ADA (the drug) between strata. A receiver operating characteristic (ROC) curve analysis was performed for serum drug levels to identify the cut-off value associated with treatment continuity. The two groups of patients were divided into a low-drug level group below the cut-off value and a high-drug level group above the cutoff value. We used the Kaplan-Meier estimator to generate survival curves for the high-drug level and the low-drug level groups and compared persistence rate with ADA between strata using the log-rank test. Next, the ROC curve analysis was performed to determine the cut-off value of serum drug level for endoscopic remission. We divided the patients into two groups, the low-drug level group below the cut-off value and the high-drug level group above the cut-off value, and compared the endoscopic remission rate between the two groups using the $\chi^{2}$ test. A univariate analysis was performed to identify factors affecting the serum ADA level via Mann-Whitney's U-test, Pearson's correlation coefficient test, and Spearman's correlation coefficient using the log-rank test. Factors included in the model were sex, age, body weight, IBD type (UC or CD), disease duration, the time from diagnosis to first ADA administration, 
the period from the start of ADA therapy to TDM implementation, IFX treatment history, and concomitant use of immunomodulators. These data were obtained when TDM was performed on the aforementioned factors. All statistical analyses were performed using EZR (Saitama Medical Center, Jichi Medical University, Saitama, Japan), which is a graphical user interface for R (The R Foundation for Statistical Computing, Vienna, Austria). Results with $P<0.05$ were considered statistically significant.

\section{Ethical considerations}

The study protocol was reviewed and approved by the Committee on the Ethics of Clinical Trials Involving Human Subjects at Hamamatsu South Hospital. In addition, the trial adhered to the principles of Good Clinical Practice and the ethical standards stipulated in the 1964 Declaration of Helsinki and its subsequent amendments. Informed consent was obtained from all patients after the purpose of the study and nature of the procedures were explained to them.

\section{Results}

\section{Patient characteristics}

Nineteen patients with UC and 33 patients with CD were enrolled in this study (Table 1). Twenty-six of the 52 patients $(50.0 \%)$ were previously treated with IFX. Twenty-seven of the 52 patients $(52.0 \%)$ received immunomodulators in combination with ADA, and three of the 52 patients $(5.8 \%)$ were treated with corticosteroids. According to the Montreal classification, we observed: (i) for UC, E1: 0 cases, E2: 4 cases (21.1\%), E3: 15 cases (78.9\%); (ii) for CD, L1: 6 cases (18.2\%), L2: 4 cases (12.1\%), L3: 23 cases (69.7\%), B1: 14 cases $(42.4 \%), B 2: 12$ cases $(36.4 \%)$, and B3: 7 cases $(21.2 \%)$. In addition, 17 patients $(51.5 \%)$ with $\mathrm{CD}$ had previously undergone enterectomy. 


\begin{tabular}{|c|c|}
\hline & $N=52$ \\
\hline UC:CD, n & 19:33 \\
\hline Male:Female, $n$ & $38: 14$ \\
\hline Age, median (IQR) & $39(17-70)$ \\
\hline Disease duration, year, median (IQR) & $10.5(1-44)$ \\
\hline Time from diagnosis to first ADA administration, year, median (IQR) & $8(0-41)$ \\
\hline Period from ADA initiation to TDM implementation, week, median (IQR) & $80(2-427)$ \\
\hline Body weight, kg, median (IQR) & $55.5(37-82)$ \\
\hline IFX treatment history, $\mathrm{n}(\%)$ & $26 / 52(50 \%-0 \%)$ \\
\hline \multicolumn{2}{|l|}{ Combination treatment } \\
\hline 5-ASA, n (\%) & $50 / 52(96.2 \%)$ \\
\hline Immunomodulators (AZA, 6-MP, n [\%]) & $27 / 52(52.0 \%)$ \\
\hline Steroid (systemic), n (\%) & $3 / 52(5.8 \%)$ \\
\hline \multicolumn{2}{|c|}{$\begin{array}{l}\text { UC, ulcerative colitis; } C D \text {, Crohn's disease; IQR, interquartile range; } A D A \text {, adalimumab; TDM, therapeutic } \\
\text { drug monitoring; IFX, infliximab; IQR, interquartile range; 5-ASA, 5-aminosalicylic acid; AZA, } \\
\text { azathioprine; 6-MP, 6-mercaptopurine. }\end{array}$} \\
\hline
\end{tabular}

\section{AAA and treatment continuity}

Here, AAA positivity was observed in 12 of the 52 patients (23.1\%): 6 of the 19 (31.6\%) patients with UC and 6 of the $33(18.2 \%)$ patients with CD were positive for AAA. We found that treatment continuity was slightly lower in the AAA-positive group than in the AAA-negative group ( $P=0.223$ by log-rank test; Fig. 1$)$. Next, we compared the endoscopic remission rate between the AAA-positive and AAA-negative groups, and no significant difference was observed between the two groups $(P=0.46$, data not shown).

\section{Adalimumab level and treatment continuity}

Based on the results of the ROC curve analysis of serum ADA level performed to identify the cut-off value associated with treatment continuity, we set the cut-off value of serum ADA level to $9.2 \mu \mathrm{g} / \mathrm{mL}$ (area under the curve $[\mathrm{AUC}]=0.767,95 \%$ confidence interval $[\mathrm{Cl}]=0.636-0.899$, positive predictive value $[\mathrm{PPV}]$ $=72 \%$, negative predictive value $[N P V]=74 \%$, sensitivity $=0.600$, specificity $=0.852$, and accuracy $=73 \%$; 
Fig. 2a). Patients with serum ADA level of $<9.2 \mu \mathrm{g} / \mathrm{mL}$ were assigned to the low-drug level group and those with $\geq 9.2 \mu \mathrm{g} / \mathrm{mL}$ to the high-drug level group. Treatment continuity was significantly higher in the high-drug level group than in the low-drug level group $(P<0.01$, log-rank test; Fig. 2b).

\section{Adalimumab level and endoscopic remission}

We analyzed the remission rate in patients who underwent endoscopy (44/52) after ADA administration. Endoscopic remission was observed in 15 of the 44 patients (34.1\%), and the serum ADA level was significantly higher in the endoscopic remission group than in the non-remission group (12.4 vs. $6.4 \mu \mathrm{g} / \mathrm{mL}, P=0.02$; Fig. 3a). An ROC curve analysis was performed to determine the optimal serum ADA level for achieving endoscopic remission, and a serum ADA level of $11.1 \mu \mathrm{g} / \mathrm{mL}$ was identified as the cutoff value $(A U C=0.716,95 \% \mathrm{Cl} 0.533-0.900, P P V=75 \%, N P V=81 \%$, sensitivity $=0.600$, specificity $=0.897$, accuracy $=80 \%$; Fig. $3 \mathrm{~b}$ ). We then compared the endoscopic remission rate between the low-drug level group with a serum ADA level of $<11.1 \mu \mathrm{g} / \mathrm{mL}$ and the high-drug level group with a serum ADA level of $\geq$ $11.1 \mu \mathrm{g} / \mathrm{mL}$. A significantly higher number of patients achieved endoscopic remission in the high-drug level group $(32 / 44,72.7 \%)$ than in the low-drug level group $(12 / 44,27.3 \%)(P<0.01)$.

\section{Relationship between patient- and disease-related factors and drug levels}

We performed a univariate analysis to identify the factors that may influence the serum ADA level (Table 2). None of the factors were found to be associated with ADA level. We also compared treatment continuity with and without immunomodulators (combination therapy vs. monotherapy) but found no significant difference between the groups ( $P=0.40$; Fig. 4). 
Sex

Age

Body weight

UC or CD $0.567^{a}$

$0.860^{b}$

$0.570^{\mathrm{b}}$

$0.430^{\mathrm{a}}$

Disease duration (years) $0.585^{c}$

Period from diagnosis to ADA start (years) $0.386^{\mathrm{C}}$

Period from ADA start to TDM implementation (weeks) $0.419^{c}$

IFX treatment history

$0.714^{\mathrm{a}}$

Combination of immunomodulators

$0.252^{\mathrm{a}}$

UC, ulcerative colitis; CD, Crohn's disease; ADA, adalimumab; TDM. therapeutic drug monitoring; IFX, infliximab

a) Mann-Whitney's U test, b) Pearson's correlation coefficient test, c) Spearman's correlation coefficient by rank test.

\section{Discussion}

In this study, we showed that the serum ADA levels, including non-trough values, but excluding values obtained within 3 days of ADA administration, reflect the subsequent clinical course and endoscopic outcome. Our study demonstrated that ADA continuity can be expected if the non-trough serum ADA level (except within 3 days of ADA administration) is $\geq 9.2 \mu \mathrm{g} / \mathrm{mL}$ and that endoscopic remission can be expected if the level is $\geq 11.1 \mu \mathrm{g} / \mathrm{mL}$. A previous study on trough level of ADA during maintenance therapy reported that $5-5.8$ and $4.9-7.1 \mu \mathrm{g} / \mathrm{mL}$ were the lower limits of the therapeutic range when clinical remission and mucosal healing were the desired targets, respectively; both limits are approximately $4 \mu \mathrm{g} / \mathrm{mL}$ lower than our non-trough level. As these are the lower limits of the therapeutic range and the variation in the levels over 2 weeks between successive ADA administrations is approximately $3 \mu \mathrm{g} / \mathrm{mL}$ [16], our results are valid.

In other studies, AAA positivity was estimated to be $12.7 \%-30.9 \%[9,11,15,17]$, which is consistent with our results in this study (23.1\%). Nakase et al. reported that AAA and clinical remission were significantly correlated in patients with $C D$ receiving ADA [18]. In contrast, Robin et al. showed that AAA positivity was higher in patients with clinically active disease than in patients with clinical remission (13.5 vs. $9.8 \mathrm{ng} / \mathrm{mL}$, respectively) [17]. In addition, AAA positivity was lower in patients who achieved mucosal 
healing than in those who did not $(6.5 \mathrm{vs} .14 .1 \mathrm{ng} / \mathrm{mL} ; P=0.06)$. Our study also showed that AAA did not correlate with treatment continuity or endoscopic outcomes; hence, the importance of measuring AAA level in clinical practice should be investigated further.

Reports on the benefits of the combined use of immunomodulators with ADA are controversial [19-24]. Although there was no association between the concomitant use of immunomodulators with ADA versus only ADA in this study $(P=0.252)$, we found that AAA positivity was significantly lower in the immunomodulator combination group (1/27 [3.7\%] vs. 11/25 [44\%], $P<0.05$, Mann-Whitney's U-test). However, there was no significant difference in treatment continuity with or without immunomodulators $(P=0.40)$. Using immunomodulators suppressed AAA production, but it did not improve any clinical outcomes in our study. However, a recent study reported that treatment continuity was higher with azathioprine when a second anti-TNFa agent was used in cases where the use of the first anti-TNFa agent resulted in immune-mediated LOR [25]. Further research is needed to determine whether it is necessary to use an immunomodulator with ADA in real-world clinical settings. In our study, because we found no other factors related to ADA level, we concluded that ADA is clinically effective if the serum ADA level is high, regardless of sex, weight, disease duration and IFX treatment history. Therefore, to predict the therapeutic efficacy of ADA, actual measurement of ADA level is important, and not the timing of blood collection to specifically measure the trough level (unless collected within 3 days of ADA administration).

A limitation of this study was that the time of measurement of ADA level differed for each patient. There are a few studies on the pharmacokinetics of ADA in patients with IBD, but information about the changes in drug level after administration is limited. A post-hoc study on the pharmacokinetics of 65 patients with $C D$ receiving $A D A$ reported that the half-life of $A D A$ was 22 days in AAA-negative patients [26]. A prospective observational study in which 19 patients with $C D$ treated with ADA underwent TDM at multiple timepoints indicated that there was negligible change in the serum ADA level over 9 days after administration [15]. Another prospective study on the pharmacokinetics of seven patients with CD undergoing remission maintenance therapy with ADA reported a minimal difference between the peak and trough blood ADA levels during ADA therapy [27]. From these studies, we inferred that the difference in the timing of TDM administration would have negligible effect on the serum ADA level in patients repeatedly receiving ADA. This is because the ADA level in the blood increases and disappears relatively slowly because of the subcutaneous formulation, and the administration interval is 2 weeks, which is shorter than other drugs.

\section{Conclusions}

In this study, we evaluated non-trough drug levels, which may be useful in predicting the outcomes of ADA therapy, unlike with other biologics. Although this was a retrospective study based on actual clinical data, our study had a long observation period: median of 102 weeks and maximum of 593 weeks. We could not find any longer-term observational study that examined the relationship between the ADA level and its therapeutic effect. 
In patients with IBD receiving ADA, a higher serum ADA level was associated with higher treatment continuity and endoscopic remission rates; however, AAA positivity did not affect ADA continuity. None of the factors we examined (such as the history of IFX administration and concomitant use of immunomodulators) were associated with serum ADA level. Therefore, to predict clinical outcomes, it would be useful to measure the blood level of ADA regardless of the timing of trough.

\section{Abbreviations}

ADA Adalimumab

AAA Anti-ADA antibody

AUC Area under the curve

CD Crohn's disease

ELISA Enzyme-linked immunosorbent assays

IBD Inflammatory bowel disease

LOR Loss of response

ROC Receiver operating characteristic

TDM Therapeutic drug monitoring

UC Ulcerative colitis

\section{Declarations}

\section{Ethics approval and consent to participate}

The study protocol was reviewed and approved by the Committee on the Ethics of Clinical Trials Involving Human Subjects at Hamamatsu South Hospital. The trial adhered to the principles of Good Clinical Practice and the ethical standards stipulated in the 1964 Declaration of Helsinki and its subsequent amendments. Informed consent was obtained from all patients.

\section{Consent of publication}

Not applicable.

\section{Availability of data and materials}

Not applicable. 


\section{Competing interests}

The authors declare that they have no competing interests.

\section{Funding}

None

\section{Author Contributions}

$\mathrm{MK}$ and $\mathrm{HH}$ were involved in the investigator and patient recruitment, data collection, and interpretation, and manuscript drafting. KS supervised the study. KS and MK contributed to the drafting of the manuscript. TM, NI, Satoshi Tamura, Shinya Tani, MY, YH, MI, SO, and TF contributed to statistical analysis and data interpretation. $\mathrm{MK}, \mathrm{HH}, \mathrm{RT}, \mathrm{AM}$, and $\mathrm{KI}$ recruited patients and recorded data. All authors contributed to the critical revision and final approval of the manuscript.

\section{Acknowledgements}

Not applicable

\section{References}

1. Abraham C, Cho JH. Inflammatory bowel disease. N Engl J Med 2009;361:2066-78.

2. Podolsky DK. Inflammatory bowel disease. N Engl J Med 2002;347:417-29.

3. Verstockt B, Ferrante M, Vermeire S, Van Assche G. New treatment options for inflammatory bowel diseases. J Gastroenterol 2018;53:585-90.

4. Paul S, Del Tedesco E, Marotte H, Rinaudo-Gaujous M, Moreau A, Phelip J-M, et al. Therapeutic drug monitoring of infliximab and mucosal healing in inflammatory bowel disease: a prospective study. Inflamm Bowel Dis 2013;19:2568-76.

5. Ungar B, Levy I, Yavne Y, Yavzori M, Picard O, Fudim E, et al. Optimizing anti-TNF-a therapy: serum levels of infliximab and adalimumab are associated with mucosal healing in patients with inflammatory bowel diseases. Clin Gastroenterol Hepatol 2016;14:550-7.

6. Papamichael K, Baert F, Tops S, Van Assche G, Rutgeerts P, Vermeire S, et al. Post-induction adalimumab concentration is associated with short-term mucosal healing in patients with ulcerative colitis. J Crohns Colitis 2017;11:53-9.

7. Gisbert JP, Panés J. Loss of response and requirement of infliximab dose intensification in Crohn's disease: a review. Am J Gastroenterol 2009;104:760-7.

8. Billioud V, Sandborn WJ, Peyrin-Biroulet L. Loss of response and need for adalimumab dose intensification in Crohn's disease: a systematic review. Am J Gastroenterol 2011;106:674-84.

9. Imaeda H, Bamba S, Takahashi K, Fujimoto T, Ban H, Tsujikawa T, et al. Relationship between serum infliximab trough levels and endoscopic activities in patients with Crohn's disease under scheduled 
maintenance treatment. J Gastroenterol 2014;49:674-82.

10. Nanda KS, Cheifetz AS, Moss AC. Impact of antibodies to infliximab on clinical outcomes and serum infliximab levels in patients with inflammatory bowel disease (IBD): a meta-analysis. Am J Gastroenterol 2013;108:40-7.

11. Mazor Y, Almog R, Kopylov U, Ben Hur D, Blatt A, Dahan A, et al. Adalimumab drug and antibody levels as predictors of clinical and laboratory response in patients with Crohn's disease. Aliment Pharmacol Ther 2014;40:620-8.

12. Mitrev N, Vande Casteele N, Seow CH, Andrews JM, Connor SJ, Moore GT, et al. Review article: Consensus statements on therapeutic drug monitoring of anti-tumour necrosis factor therapy in inflammatory bowel diseases. Aliment Pharmacol Ther 2017;46:1037-53.

13. Negoescu DM, Enns EA, Swanhorst B, Baumgartner B, Campbell JP, Osterman MT, et al. Proactive vs reactive therapeutic drug monitoring of infliximab in Crohn's disease: a cost-effectiveness analysis in a simulated cohort. Inflamm Bowel Dis 2020;26:103-11.

14. Peyrin-Biroulet L, Sandborn W, Sands BE, Reinisch W, Bemelman W, Bryant RV, et al. Selecting therapeutic targets in inflammatory bowel disease (STRIDE): determining therapeutic goals for treatto-target. Am J Gastroenterol 2015;110:1324-38.

15. Ward MG, Thwaites PA, Beswick L, Hogg J, Rosella G, Van Langenberg D, et al. Intra-patient variability in adalimumab drug levels within and between cycles in Crohn's disease. Aliment Pharmacol Ther 2017;45:1135-45.

16. Gibson DJ, Ward MG, Rentsch C, Friedman AB, Taylor KM, Sparrow MP, et al. Review article: Determination of the therapeutic range for therapeutic drug monitoring of adalimumab and infliximab in patients with inflammatory bowel disease. Aliment Pharmacol Ther 2020;51:612-28.

17. Roblin X, Marotte H, Rinaudo M, Del Tedesco E, Moreau A, Phelip JM, et al. Association between pharmacokinetics of adalimumab and mucosal healing in patients with inflammatory bowel diseases. Clin Gastroenterol Hepatol 2014;12:80-4.

18. Nakase H, Motoya S, Matsumoto T, Watanabe K, Hisamatsu T, Yoshimura N, et al. Significance of measurement of serum trough level and anti-drug antibody of adalimumab as personalised pharmacokinetics in patients with Crohn's disease: a subanalysis of the DIAMOND trial. Aliment Pharmacol Ther 2017;46:873-82.

19. Peters CP, Eshuis EJ, Toxopeüs FM, Hellemons ME, Jansen JM, D'Haens GRAM, et al. Adalimumab for Crohn's disease: Long-term sustained benefit in a population-based cohort of 438 patients. J Crohn Colitis 2014;8:866-75.

20. Khanna R, Bressler B, Levesque BG, Zou G, Stitt LW, Greenberg GR, et al. Early combined immunosuppression for the management of Crohn's disease (REACT): a cluster randomised controlled trial. Lancet 2015;386:1825-34.

21. Reenaers C, Louis E, Belaiche J, Seidel L, Keshav S, Travis S. Does co-treatment with immunosuppressors improve outcome in patients with Crohn's disease treated with adalimumab? Aliment Pharmacol Ther 2012;36:1040-8. 
22. Matsumoto T, Motoya S, Watanabe K, Hisamatsu T, Nakase H, Yoshimura N, et al. Adalimumab monotherapy and a combination with azathioprine for Crohn's disease: a prospective, randomized trial. J Crohns Colitis 2016;10:1259-66.

23. Matar M, Shamir R, Turner D, Broide E, Weiss B, Ledder O, et al. Combination therapy of adalimumab with an immunomodulator is not more effective than adalimumab monotherapy in children with Crohn's disease: a post hoc analysis of the PAILOT randomized controlled trial. Inflamm Bowel Dis. DOl: 10.1093/ibd/izz294.

24. Narula N, Kainz S, Petritsch W, Haas T, Feichtenschlager T, Novacek G, et al. The efficacy and safety of either infliximab or adalimumab in 362 patients with anti-TNF-a naïve Crohn's disease. Aliment Pharmacol Ther 2016;44:170-80.

25. Roblin X, Williet N, Boschetti G, Phelip J-M, Del Tedesco E, Berger A-E, et al. Addition of azathioprine to the switch of anti-TNF in patients with IBD in clinical relapse with undetectable anti-TNF trough levels and antidrug antibodies: a prospective randomised trial. Gut 2020;69:1206-12.

26. Ternant D, Karmiris K, Vermeire S, Desvignes C, Azzopardi N, Bejan-Angoulvant T, et al. Pharmacokinetics of adalimumab in Crohn's disease. Eur J Clin Pharmacol 2015;71:1155-7.

27. Stewart MJ, Dubinsky M, Morganstern B, Vasiliauskas E, Ippoliti A, Shih DQ, et al. Sa1965 the steadystate pharmacokinetics of adalimumab: do we need to drink from the "trough?" Gastroenterology 2016;150:S418-9.

\section{Figures}




\section{Figure 1}

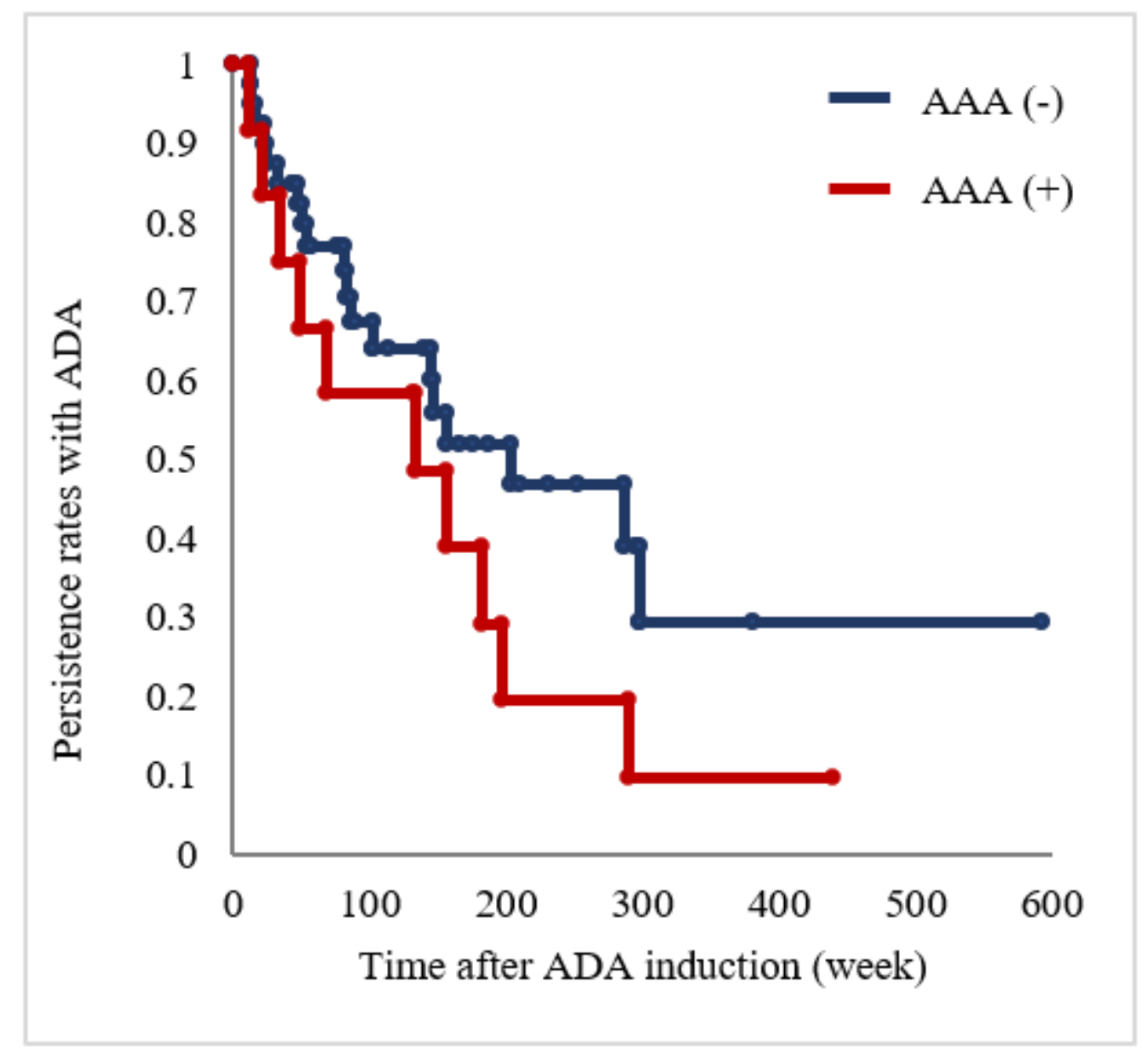

Figure 1

Kaplan-Meier curves of time to ADA discontinuation in patients in the AAA-negative group vs. those in the AAA-positive group ( $p=0.023$ by log-rank test). ADA, adalimumab; AAA, anti-adalimumab antibody. 
Figure 2

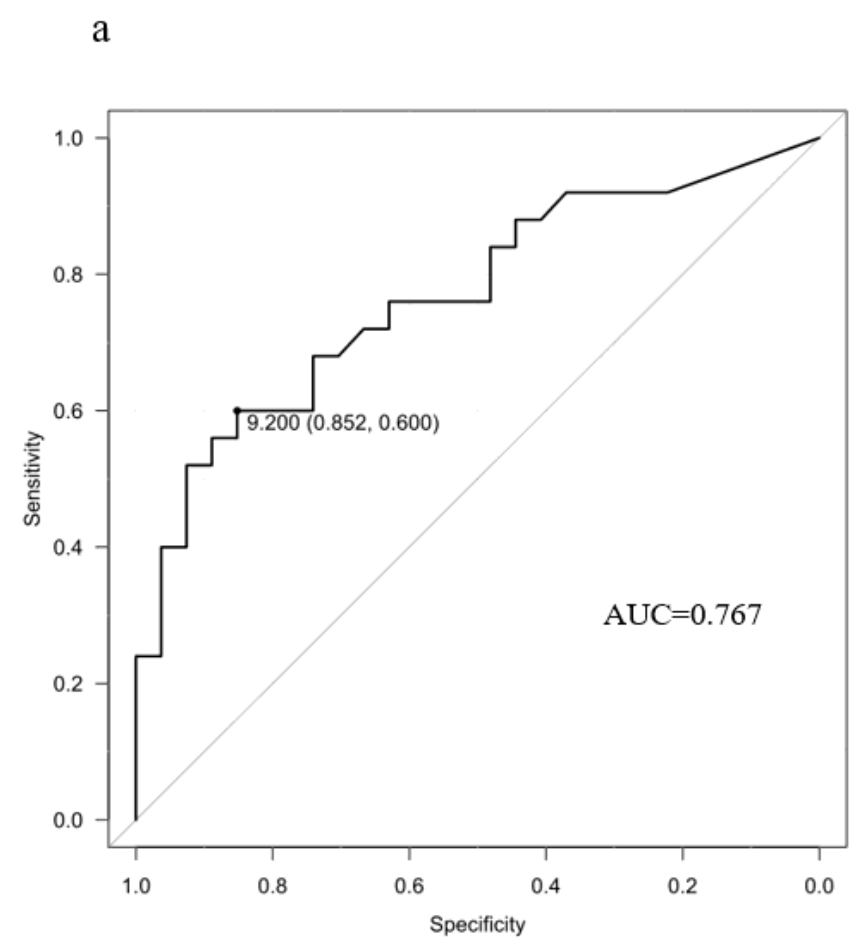

$\mathrm{b}$

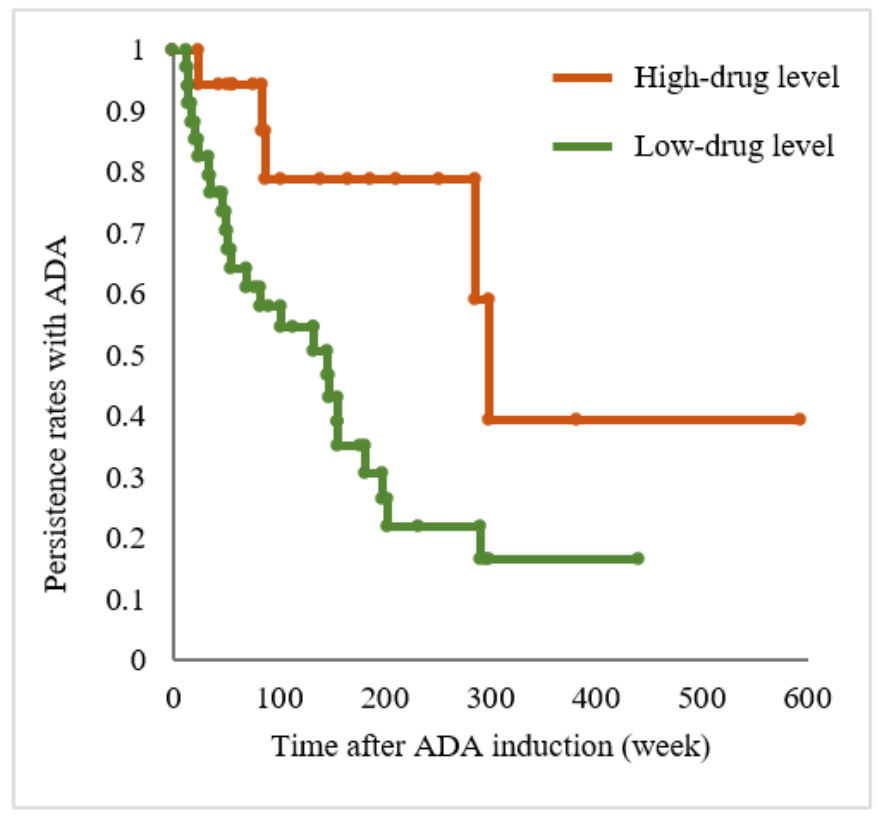

Figure 2

a. Receiver operating characteristic analysis for ADA level stratifying patients with and without ADA discontinuation. AUC, area under the curve. b. Kaplan-Meier curves of time to ADA discontinuation in patients in the high-drug level (ADA level $\geq 9.2 \mu \mathrm{g} / \mathrm{mL}$ ) group vs. low-drug level (ADA level $<9.2 \mu \mathrm{g} / \mathrm{mL}$ ) group $(P<0.01$ by log-rank test). ADA, adalimumab; $A U C$, area under the curve. 
Figure 3

a

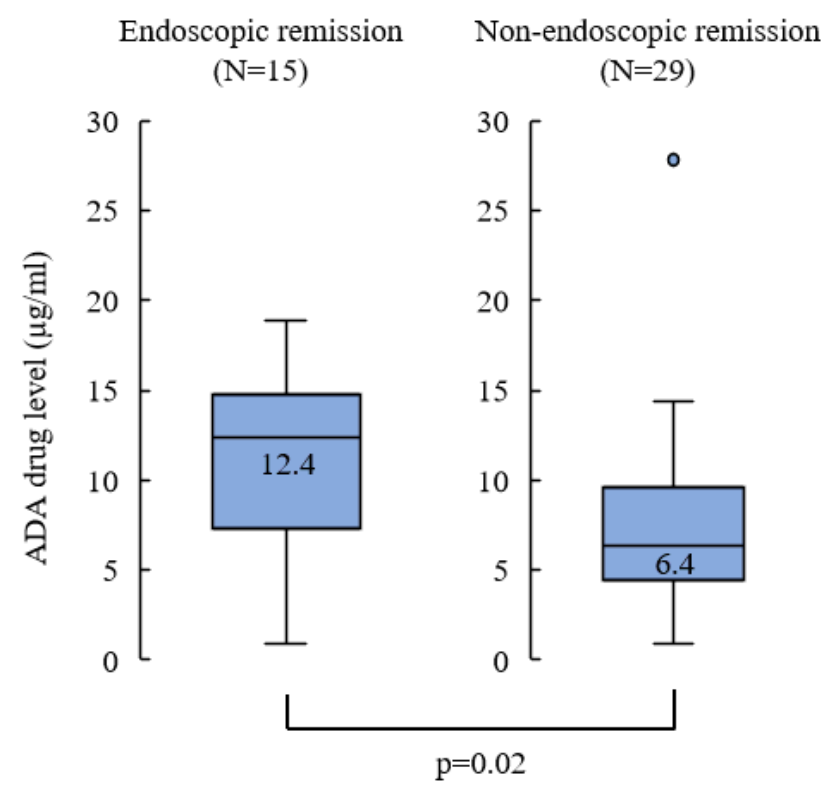

b

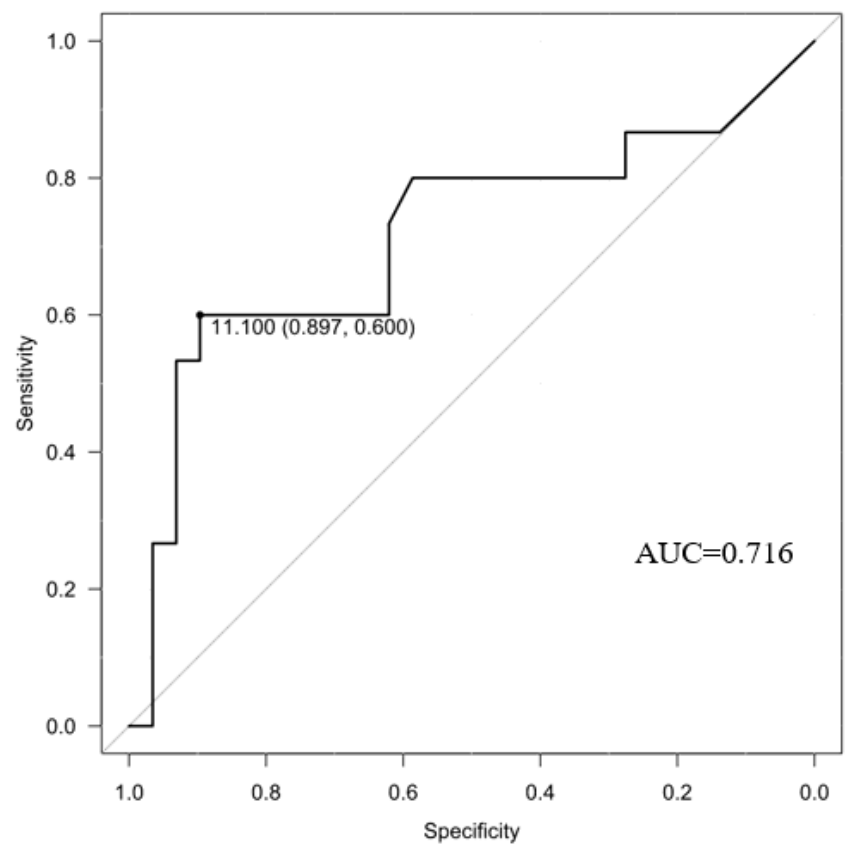

\section{Figure 3}

a. Distribution of ADA levels in patients with and without endoscopic remission. Box plots (5\%-95\%) show the median (solid line within the box), interquartile range (upper and lower box boundaries), and standard deviation (whiskers). There was a significant difference in the ADA level between patients with and without endoscopic remission ( $P=0.02$ by Mann-Whitney's U-test). b. Receiver operating characteristic curve analysis for ADA level stratifying patients with and without endoscopic remission. AUC, area under the curve; ADA, adalimumab. 
Figure 4

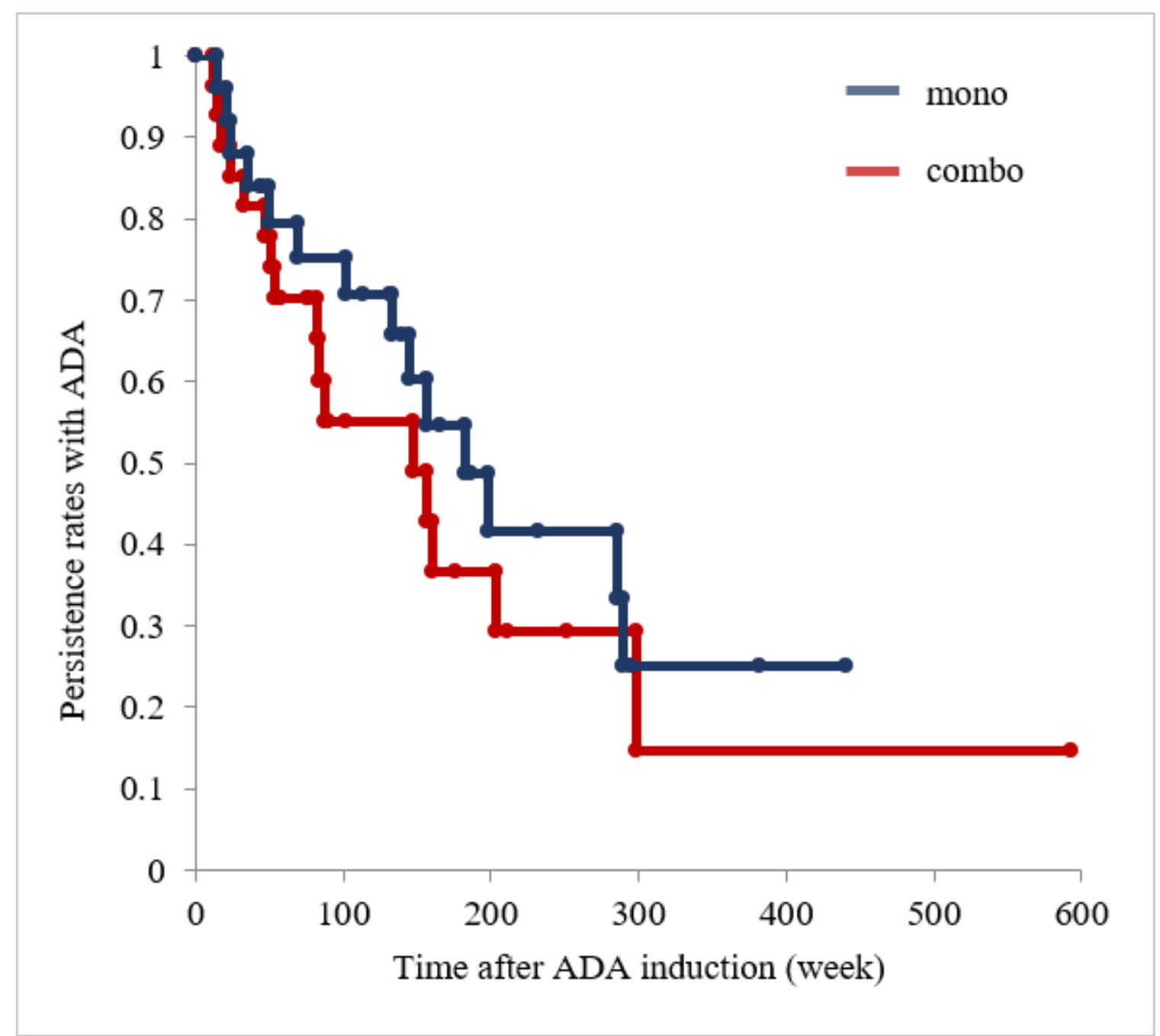

Figure 4

Kaplan-Meier curves of time to ADA discontinuation in patients in the ADA monotherapy group vs. those in the ADA plus immunomodulator combination therapy group $(P=0.40$ by log-rank test). Mono, monotherapy; combo, combination therapy; ADA, adalimumab. 